\title{
Hormone gene signature guides a novel therapeutic opportunity to improve sensitivity to HER family inhibition in lung cancer
}

\author{
Zachary A. Yochum ${ }^{1,2}$, Laura P. Stabile ${ }^{3 \wedge}$ \\ ${ }^{1}$ Department of Medicine, Division of Hematology-Oncology, UPMC Hillman Cancer Center, Pittsburgh, PA, USA; ${ }^{2}$ Medical Scientist Training \\ Program, University of Pittsburgh School of Medicine, Pittsburgh, PA, USA; ${ }^{3}$ Department of Pharmacology \& Chemical Biology, University of \\ Pittsburgh School of Medicine, UPMC Hillman Cancer Center, Pittsburgh, PA, USA \\ Correspondence to: Laura P. Stabile, PhD. UPMC Hillman Cancer Center, Hillman Cancer Center Research Pavilion, Suite 2.18d Lab: 2.7, 5117 \\ Centre Avenue, Pittsburgh, PA 15213-1863, USA. Email: stabilela@upmc.edu. \\ Provenance and Peer Review: This article was commissioned by the editorial office, Translational Lung Cancer Research. The article did not undergo \\ external peer review. \\ Comment on: Almotlak AA, Farooqui M, Siegfried JM. Inhibiting Pathways Predicted from a Steroid Hormone Gene Signature Yields Synergistic \\ Antitumor Effects in NSCLC. J Thorac Oncol 2020;15:62-79.
}

Submitted Apr 30, 2020. Accepted for publication May 14, 2020.

doi: 10.21037/tlcr-20-617

View this article at: http://dx.doi.org/10.21037/tlcr-20-617

Lung cancer is the top cause of cancer-related deaths for both men and women in the United States and worldwide (1). Survival rates remain poor for lung cancer patients as it is typically diagnosed at an advanced stage and there are few effective therapies for metastatic disease. However, there have been advances in the treatment of patients with advanced non-small cell lung cancer (NSCLC), which accounts for approximately $80-85 \%$ of all lung cancers, stemming from the introduction of targeted therapy and immunotherapy (2). Patients diagnosed with NSCLC are classified into subgroups based on the presence of molecular drivers. Approximately 50\% of patients have an identifiable molecular driver, some of which have FDA-approved targeted therapies $(3,4)$. The success of targeted therapies is highlighted by the $15 \%$ of NSCLC patients with EGFR mutations that are sensitive to EGFR tyrosine kinase inhibitors (TKIs) (3-5). In EGFR-mutant NSCLC patients, EGFR TKIs provide superior objective response rates (ORR) and progressionfree survival (PFS) when compared to chemotherapy (5). Osimertinib, a third-generation EGFR TKI with activity against multiple EGFR mutations, including the "gatekeeper" T790M mutation which renders firstgeneration TKIs ineffective, is now the first-line treatment for EGFR-mutant NSCLC patients as it improves PFS and overall survival (OS) when compared to firstgeneration EGFR TKIs (6). In patients without targetable molecular drivers, immunotherapy, specifically the PDL1 antibody, pembrolizumab, has become the standardof-care. Multiple phase III trials have demonstrated that pembrolizumab whether as a single-agent (for tumors with $\mathrm{PD}-\mathrm{L} 1$ positivity $\geq 50 \%$ ) or in combination with chemotherapy provides superior PFS and OS when compared to chemotherapy in metastatic NSCLC patients $(7,8)$. Despite the promise of these treatment modalities, the frequency of acquired and de novo therapeutic resistance has limited their long-term efficacy.

The estrogen pathway has emerged as a potential target in lung cancer. Estrogen receptors (ERs) and aromatase are expressed in NSCLCs (9). Of the two types of ERs (ER $\alpha$ and $\mathrm{ER} \beta), \mathrm{ER} \alpha$ is the major driver of breast tumorigenesis, while ER $\beta$ appears to play a more important role in NSCLC tumorigenesis as it more frequently expressed and expressed at higher levels than ER $\alpha$ (10). Initial evidence supporting the role of estrogen signaling in lung cancer tumorigenesis stems from population studies showing that women using hormone replacement therapy had increased rates of lung cancer incidence and mortality $(11,12)$.

^ ORCID: Zachary A. Yochum: 0000-0001-6483-8089; Laura P. Stabile: 0000-0002-1822-2707. 
Conversely, longitudinal data on women with ER+ breast cancers treated with anti-estrogen therapies demonstrated that these patients had a decreased risk of developing lung cancer (13). Preclinically, exogenous $\beta$-estradiol promotes NSCLC cell proliferation via increased transcription of estrogen-responsive genes and non-genomic activation of mitogenic pathways $(9,14)$. Targeting the estrogen signaling pathway with aromatase inhibitors and/or fulvestrant, a selective estrogen receptor degrader, has anti-proliferative effects in vitro and impairs carcinogen-induced lung tumorigenesis in murine models $(9,15)$.

Clinical trials investigating estrogen targeted therapy in lung cancer have largely stemmed from preclinical evidence demonstrating crosstalk between the ER and EGFR signaling pathways. Previous studies have revealed that estrogen signaling regulates the expression of EGFR and EGFR signaling reciprocally regulates the expression of ER in NSCLC cells. Additionally, $\beta$-estradiol causes rapid activation of EGFR and downstream pathways (9). The combination of first-generation EGFR TKI, gefitinib, and fulvestrant inhibits NSCLC tumor growth and induces apoptosis both in vitro and in vivo (9). Based on this preclinical evidence, there have been multiple clinical trials investigating the efficacy of combining fulvestrant and EGFR TKIs in NSCLC. An initial phase II trial compared the combination of fulvestrant and erlotinib versus singleagent erlotinib in NSCLC patients (16). While there was no significant difference in ORR, PFS, or OS between the combination and erlotinib alone, a subset analysis revealed EGFR wild-type (WT) patients that received the combination had a significantly increased PFS and OS compared to erlotinib alone (16). A recent follow-up phase II trial evaluated this combination in female patients with metastatic NSCLC. Patients were separated into EGFR-mutant and WT subgroups and randomized to receive single-agent first-generation EGFR TKI or a firstgeneration EGFR TKI in combination with fulvestrant. While the combination was well-tolerated, the addition of fulvestrant did not extend PFS or OS in either the EGFR-mutant or WT cohorts (17). Regardless of EGFR status or treatment group, ER $\alpha$ expression was associated with a trend toward increased PFS and OS suggesting $\mathrm{ER} \alpha$ expression may be a prognostic marker rather than predictor of response to fulvestrant in NSCLC (17).

In addition to being a potential therapeutic target, estrogen signaling also has prognostic significance in NSCLC. Previous studies have shown an association between ER subtypes and aromatase expression with
NSCLC survival (10). Additionally, the prediction analysis of microarray 50 (PAM50) gene signature, which is frequently used to subtype breast cancers and predict survival in ER+ breast cancer (18), has prognostic significance in NSCLC as demonstrated in three cohorts: a stage I NSCLC cohort, The Cancer Genome Atlas (TCGA) data for NSCLC, and an ER $\beta$ + all stage NSCLC cohort (19). Interestingly, cross-validation of the PAM50 gene signature in the Stage I cohort revealed that a group of seven genes was most significantly associated with survival and this group was prognostic both in the Stage I cohort and in a TCGA squamous cohort (19). This seven gene crossvalidation group included a known ER target gene, $P G R$, and pathway analysis implicated this group in estrogen and HER2/HER3 signaling (19). Furthermore, ER $\beta$ protein expression positively correlated with HER2 expression in tumor tissue (19).

Given the aforementioned study demonstrated that the ER and HER2/3 signaling network has prognostic significance lung cancer, a recent study reported in the Fournal of Thoracic Oncology by Almotlak et al. explored the efficacy of targeting this pathway in NSCLC. The authors demonstrated that the combination of fulvestrant and dacomitinib, a pan-HER TKI, has synergistic activity in multiple ER $\beta+$ NSCLC cell lines, including cells with KRAS and EGFR mutations (20). Combination treatment decreased EGFR, HER2, and HER3 activation as well as activation of canonical HER signaling pathways such as MAPK and AKT (20). Interestingly, single-agent fulvestrant increased expression of HER ligands, NRG1- $\beta 1$ and AREG, while single-agent dacomitinib led to a significant increase in aromatase and ER $\beta$ expression (Figure 1). These compensatory increases in estrogen and HER signaling were abrogated with combination therapy (20).

The authors demonstrated that this combination resulted in downregulation of phospho-cyclic adenosine monophosphate response element binding protein (p-CREB) and activator protein-1 (AP-1), transcription factors implicated in HER2 and ER signaling (Figure 1) (20). Additionally, utilizing the sevengene PAM50 panel established in the aforementioned study (19), the authors demonstrated that dacomitinib and fulvestrant treatment reversed the gene signature associated with poor survival (Figure 1) (20). This gene signature reversal was recapitulated with an $\mathrm{AP}-1$ inhibitor and partially recapitulated with a p-CREB inhibitor implicating downregulation of these two transcription factors as potential targets of the fulvestrant and dacomitinib 


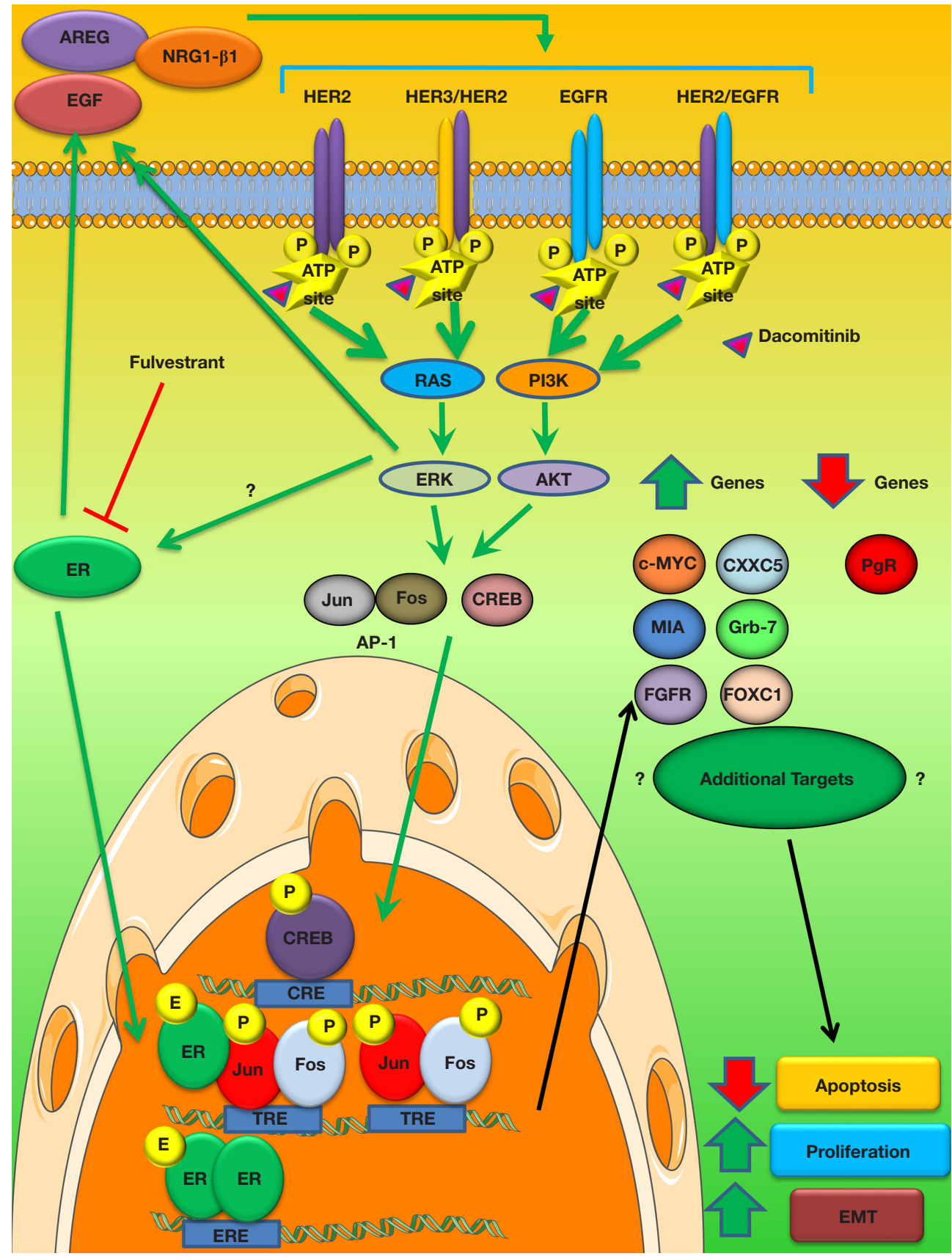

Figure 1 Estrogen receptor (ER) and pan-HER signaling inhibition has synergistic activity and leads to gene expression changes associated with good prognosis in non-small cell lung cancer (NSCLC). In the discussed report, the authors demonstrate that fulvestrant and dacomitinib, a pan-HER family inhibitor, has synergistic activity in NSCLC including tumors without effective therapies such as those with $K R A S$ mutations. Interestingly, the authors demonstrate that single-agent fulvestrant or dacomitinib led to upregulation of the other pathway. ER upregulates HER signaling through increasing expression of HER ligands, while it remains largely unclear how ER expression is upregulated by HER signaling. This reciprocal upregulation was prevented with combination therapy. The activity of this combination was associated with downregulation of the activator protein-1 (AP-1) and cAMP response element binding protein (CREB) transcription factors and reversal of a seven-gene signature previously implicated in poor prognosis in NSCLC. Future studies are needed to determine whether there are additional molecular targets of this combination, which genes/ pathways are required for activity of the combination, and the mechanisms by which this combination leads to synergistic activity in NSCLC. A portion of this figure was created using modified Servier Medical Art templates, which are under a Creative Commons Attribution 3.0 Unported License: https://smart.servier.com. E, estrogen; ERE, estrogen response element; TRE, TPA response element; CRE, cAMP response element; EMT, epithelial-mesenchymal transition. 
combination (20).

Combination ER and pan-HER inhibition also had activity in vivo. In xenograft mouse models of EGFR/KRAS WT and KRAS-mutant NSCLC, combination therapy significantly inhibited tumor growth and led to regression of most tumors (20). Paralleling their in vitro studies, the authors demonstrated that this combination in vivo led to decreased phospho-EGFR levels, decreased total HER2 and HER3 expression, decreased phospho-HER2/ phospho-HER3 dimer formation, and reversed the gene signature associated with poor survival in the seven-gene PAM50 panel (20) Additionally, using an EGFR-mutant (E746-A7504) NSCLC xenograft model, both single-agent dacomitinib and the combination treatment led to complete tumor regression (20). Given the complete response in both groups, treatments were stopped and recurrence was monitored. While the combination treatment did not significantly extend survival, the tumors that recurred in the combination group were histologically less aggressive when compared to the dacomitinib group (20). The authors demonstrated the tumors that recurred in the combination group had decreased stem cell markers, MET signaling, and AKT signaling which may account for their less aggressive phenotype (20).

While subsets of NSCLC patients benefit from targeted therapies, there remains a significant patient population without an identifiable molecular driver ( $40-50 \%)$ and the most common molecular driver, mutant KRAS currently does not have effective targeted therapy (3). A strength of Almotlak and colleagues recent study is that the authors demonstrate that the combination of fulvestrant and dacomitinib has synergistic activity both in vitro and in vivo in $W T$ and KRAS-mutant NSCLC (20). Their work suggests that this combination may be a treatment strategy in the NSCLC population without an efficacious targeted therapy. Previous studies have demonstrated that ER $\beta$ can lead to EGFR TKI resistance (21). This is the first study demonstrating that targeting estrogen signaling can improve the efficacy of an EGFR TKI in EGFR-mutant NSCLC (20). An additional strength of this study is that the authors demonstrate that fulvestrant not only has synergistic activity with EGFR TKIs in vitro but also can delay resistance to EGFR TKIs in vivo (20). This work suggests that fulvestrant and dacomitinib may be effective in NSCLC without targetable alterations and improve responses in patients whose tumors have targetable alterations with approved therapies. Furthermore, this combination may lend itself to an accelerated translation into the clinic given that both fulvestrant and dacomitinib are FDA-approved therapies with well-established safety profiles.

Despite demonstrating broad efficacy of the combination of fulvestrant and dacomitinib, the mechanism(s) by which this combination leads to anti-tumor activity is still unclear. The combination of dacomitinib and fulvestrant downregulated AP-1, p-CREB, ERK, and AKT signaling as well as modulated the seven genes identified in the PAM50 panel (20) (Figure 1). Future studies are needed to identify whether additional pathways and/or genes are modulated by this combination and which of these pathways/molecules is required for response to this combination (Figure 1). Induction of apoptosis and suppression of proliferation was observed with dacomitinib and fulvestrant treatment. Additional studies are needed to determine if the activity of this combination requires apoptosis and/or suppression of proliferation or if other mechanisms drive response, such as cell cycle arrest. Estrogen and HER signaling have also been implicated in epithelial-mesenchymal transition (EMT) in breast and lung cancer $(22,23)$. Future studies should determine if reversing EMT is required for response to dacomitinib and fulvestrant. Determining the mechanism(s) of action may result in the identification of potential biomarkers of response and additional therapeutic targets. Additionally, while the authors demonstrated that the combination of dacomitinib and fulvestrant is efficacious in WT and KRAS-mutant NSCLC, given the molecular and genetic heterogeneity within these subtypes, dacomitinib and fulvestrant are unlikely to be broadly efficacious in these groups. Future studies are needed both to test this combination in additional WT and KRAS-mutant NSCLC models and to determine how genetic and molecular backgrounds, such as p53 status and ER $\beta$ expression levels, influence response.

A weakness of this study is that it does not establish whether the activity of the fulvestrant and dacomitinib combination requires pan-HER inhibition. Given that dacomitinib irreversibly inhibits EGFR, HER2, and HER4, future studies are needed to determine if the efficacy of this combination requires inhibition of specific HER family members or pan-HER inhibition. The genetic background of NSCLC will likely determine if inhibition of individual HER family members versus pan-HER inhibition is required. For example, KRASmutant NSCLC tumors are only susceptible to pan-HER inhibition, as EGFR inhibition alone causes rapid resistance through the upregulation of alternative HER family 
members (24). While there is some evidence to suggest that WT NSCLC patients may be susceptible to EGFR TKIs in combination with fulvestrant $(9,16)$, the recent LADIE trial demonstrated first-generation EGFR TKIs in combination with fulvestrant are not efficacious in this population (17). There have been multiple studies, including Almotlak and colleagues recent study, that suggest that targeting HER2/ER crosstalk may be a more relevant therapeutic target in WT NSCLC patients $(19,20)$. It is important to determine in WT NSCLC if the efficacy of dacomitinib plus fulvestrant requires dual inhibition of EGFR and HER2 or HER2 alone. If EGFR and HER2 co-inhibition is important, first-generation and third-generation EGFR TKIs are unlikely to have efficacy as they have limited activity against HER2 or WT EGFR, respectively. While second-generation EGFR TKIs, such as dacomitinib, which inhibit both WT EGFR and HER2, will be viable therapies. If HER2 inhibition alone proves to be the driver of response to dacomitinib and fulvestrant, future studies should investigate the efficacy of combining HER2-specific therapies, like trastuzumab, in combination with fulvestrant.

While dacomitinib is an approved first-line therapy for EGFR-mutant NSCLC, there have not been head-to-head comparisons of dacomitinib and the currently recommended first-line agent, osimertinib (25). Dacomitinib is unlikely to replace osimertinib as a first-line agent as it has an inferior toxicity profile and has limited activity against $T 790 M$ EGFR mutations (25). Future studies are needed to not only determine the efficacy of fulvestrant in combination with osimertinib in EGFR-mutant NSCLC but also if fulvestrant can delay or overcome osimertinib resistance. While the mechanism(s) by which fulvestrant delays dacomitinib resistance in vivo is unclear, the authors demonstrate that the combination decreases expression of stem-cell markers and the receptor tyrosine kinase, MET (20). Investigation into the mechanism(s) by which estrogen signaling may lead to EGFR TKI resistance is important as it may lead to additional therapeutic targets/strategies.

Future studies should also be aimed at investigating targeting the HER family and ER in other subsets of oncogene-driven NSCLC. Specifically, given the crosstalk between the ER and HER2 signaling pathways, fulvestrant and dacomitinib may be a potential therapeutic regimen in HER2-driven NSCLC. Approximately 5-6\% of NSCLC patients have HER2 mutations/amplification, however, this subset of patients lacks an FDA approved first-line HER2 targeted therapy (26). Additionally, HER2 amplification is a known mechanism of resistance to osimertinib in EGFR- mutant NSCLC (27). The combination of fulvestrant and dacomitinib may provide a novel therapeutic strategy for not only HER2-driven NSCLC but also HER2-mediated therapeutic resistance.

While the authors demonstrate that dacomitinib and fulvestrant have activity in xenograft models, emerging evidence suggests that response to TKIs is associated with modulation of the tumor microenvironment (TME) and recruitment of immune cells (28). Moreover, estrogen is known to promote an immunosuppressive role in the TME and recent clinical evidence suggests that estrogen signaling impairs response to immunotherapy in lung cancer patients. Analysis of clinical trials comparing singleagent immunotherapy with chemotherapy has revealed that female NSCLC patients are less likely to benefit from immunotherapy compared to male patients $(29,30)$. These sex differences suggest that hormone signaling may dampen response to immunotherapy. ERs are expressed in most immune cell types and estrogen signaling in TME has been associated with increased infiltration of tumorassociated macrophages, blunted CD8+ T cell function, increased regulatory $\mathrm{T}$ cell function, and upregulation of PD-1 and PD-L1 expression [reviewed in (31)]. Interestingly, fulvestrant was identified in a drug screening assay to enhance NSCLC cell susceptibility to immune cell cytotoxicity (32). Additionally, there is evidence in HER2 + breast cancers that the combination of HER2 targeted therapies and immunotherapy are synergistic in preclinical models (33). Future studies are needed to investigate whether targeting estrogen signaling and the HER family can improve responses to immunotherapy in immunocompetent murine models of NSCLC, including transgenic murine models of oncogene-driven NSCLC and tobacco carcinogen-induced NSCLC models.

In summary, Almotlak et al. demonstrated that the combination of fulvestrant and dacomitinib has activity in ER $\beta+$ NSCLC (20). This combination showed efficacy in NSCLC tumors with targetable oncogenic drivers such as mutant $E G F R$ and without targetable drivers such as mutant KRAS (20). Additionally, response to this combination led to a gene expression profile associated with a favorable prognosis in NSCLC (20). This study provides renewed interest in fulvestrant for NSCLC therapy and suggests that targeting ER-HER2 crosstalk may be more clinically relevant than co-targeting ER and EGFR, which could be the reason for the negative results reported in the recent LADIE trial. Further investigation into combining ER and HER targeted therapy is warranted, not only as a novel 
therapeutic strategy for subsets of NSCLC patients without effective first-line therapies but also as a strategy to improve responses to targeted therapy and immunotherapy in NSCLC. This combination may be particularly effective in female NSCLC patients, a population with known reduced efficacy to immunotherapy.

\section{Acknowledgments}

Funding: None.

\section{Footnote}

Conflicts of Interest: Both authors have completed the ICMJE uniform disclosure form (available at http://dx.doi. org/10.21037/tlcr-20-617). The authors have no conflicts of interests to declare.

Ethical Statement: The authors are accountable for all aspects of the work in ensuring that questions related to the accuracy or integrity of any part of the work are appropriately investigated and resolved.

Open Access Statement: This is an Open Access article distributed in accordance with the Creative Commons Attribution-NonCommercial-NoDerivs 4.0 International License (CC BY-NC-ND 4.0), which permits the noncommercial replication and distribution of the article with the strict proviso that no changes or edits are made and the original work is properly cited (including links to both the formal publication through the relevant DOI and the license). See: https://creativecommons.org/licenses/by-nc-nd/4.0/.

\section{References}

1. Torre LA, Siegel RL, Jemal A. Lung Cancer Statistics. Adv Exp Med Biol 2016;893:1-19.

2. Molina JR, Yang P, Cassivi SD, et al. Non-small cell lung cancer: epidemiology, risk factors, treatment, and survivorship. Mayo Clin Proc 2008;83:584-94.

3. Barlesi F, Mazieres J, Merlio JP, et al. Routine molecular profiling of patients with advanced non-small-cell lung cancer: results of a 1-year nationwide programme of the French Cooperative Thoracic Intergroup (IFCT). Lancet 2016;387:1415-26.

4. Kris MG, Johnson BE, Berry LD, et al. Using Multiplexed Assays of Oncogenic Drivers in Lung Cancers to Select Targeted Drugs. JAMA 2014;311:1998-2006.
5. Wu YL, Zhou C, Liam CK, et al. First-line erlotinib versus gemcitabine/cisplatin in patients with advanced EGFR mutation-positive non-small-cell lung cancer: analyses from the phase III, randomized, open-label, ENSURE study. Ann Oncol 2015;26:1883-9.

6. Soria JC, Ohe Y, Vansteenkiste J, et al. Osimertinib in Untreated EGFR-Mutated Advanced Non-Small-Cell Lung Cancer. N Engl J Med 2018;378:113-25.

7. Reck M, Rodriguez-Abreu D, Robinson AG, et al. Pembrolizumab versus Chemotherapy for PD-L1Positive Non-Small-Cell Lung Cancer. N Engl J Med 2016;375:1823-33.

8. Gandhi L, Rodriguez-Abreu D, Gadgeel S, et al. Pembrolizumab plus Chemotherapy in Metastatic Non-Small-Cell Lung Cancer. N Engl J Med 2018;378:2078-92.

9. Stabile LP, Lyker JS, Gubish CT, et al. Combined targeting of the estrogen receptor and the epidermal growth factor receptor in non-small cell lung cancer shows enhanced antiproliferative effects. Cancer Res 2005;65:1459-70.

10. Burns TF, Stabile LP. Targeting the estrogen pathway for the treatment and prevention of lung cancer. Lung Cancer Manag 2014;3:43-52.

11. Slatore CG, Chien JW, Au DH, et al. Lung cancer and hormone replacement therapy: association in the vitamins and lifestyle study. J Clin Oncol 2010;28:1540-6.

12. Chlebowski RT, Anderson GL, Manson JE, et al. Lung cancer among postmenopausal women treated with estrogen alone in the women's health initiative randomized trial. Journal of the National Cancer Institute 2010;102:1413-21.

13. Rosell J, Nordenskjold B, Bengtsson NO, et al. Longterm effects on the incidence of second primary cancers in a randomized trial of two and five years of adjuvant tamoxifen. Acta Oncol 2017;56:614-7.

14. Hershberger PA, Stabile LP, Kanterewicz B, et al. Estrogen receptor beta (ERbeta) subtype-specific ligands increase transcription, $\mathrm{p} 44 / \mathrm{p} 42$ mitogen activated protein kinase (MAPK) activation and growth in human nonsmall cell lung cancer cells. J Steroid Biochem Mol Biol 2009;116:102-9.

15. Stabile LP, Rothstein ME, Cunningham DE, et al. Prevention of tobacco carcinogen-induced lung cancer in female mice using antiestrogens. Carcinogenesis 2012;33:2181-9.

16. Garon EB, Siegfried JM, Stabile LP, et al. Randomized phase II study of fulvestrant and erlotinib compared with 
erlotinib alone in patients with advanced or metastatic non-small cell lung cancer. Lung Cancer 2018;123:91-8.

17. Mazieres J, Barlesi F, Rouquette I, et al. Randomized phase II trial evaluating treatment with EGFR-TKI associated with anti-estrogen in women with non-squamous advanced stage NSCLC: IFCT-1003 LADIE trial. Clin Cancer Res 2020;26:3172-81.

18. Dowsett M, Sestak I, Lopez-Knowles E, et al. Comparison of PAM50 risk of recurrence score with oncotype DX and IHC4 for predicting risk of distant recurrence after endocrine therapy. J Clin Oncol 2013;31:2783-90.

19. Siegfried JM, Lin Y, Diergaarde B, et al. Expression of PAM50 Genes in Lung Cancer: Evidence that Interactions between Hormone Receptors and HER2/HER3 Contribute to Poor Outcome. Neoplasia 2015;17:817-25.

20. Almotlak AA, Farooqui M, Siegfried JM. Inhibiting Pathways Predicted From a Steroid Hormone Gene Signature Yields Synergistic Antitumor Effects in NSCLC. J Thorac Oncol 2020;15:62-79.

21. Fu S, Liu C, Huang Q, et al. Estrogen receptor beta1 activation accelerates resistance to epidermal growth factor receptor-tyrosine kinase inhibitors in non-small cell lung cancer. Oncol Rep 2018;39:1313-21.

22. Ingthorsson S, Andersen K, Hilmarsdottir B, et al. HER2 induced EMT and tumorigenicity in breast epithelial progenitor cells is inhibited by coexpression of EGFR. Oncogene 2016;35:4244-55.

23. Zhao G, Nie Y, Lv M, et al. ERbeta-mediated estradiol enhances epithelial mesenchymal transition of lung adenocarcinoma through increasing transcription of midkine. Mol Endocrinol 2012;26:1304-15.

24. Moll HP, Pranz K, Musteanu M, et al. Afatinib restrains K-RAS-driven lung tumorigenesis. Sci Transl Med

Cite this article as: Yochum ZA, Stabile LP. Hormone gene signature guides a novel therapeutic opportunity to improve sensitivity to HER family inhibition in lung cancer. Transl Lung Cancer Res 2020;9(4):1599-1605. doi: 10.21037/tlcr-20-617
2018;10.

25. Lavacchi D, Mazzoni F, Giaccone G. Clinical evaluation of dacomitinib for the treatment of metastatic non-small cell lung cancer (NSCLC): current perspectives. Drug Des Devel Ther 2019;13:3187-98.

26. Li BT, Ross DS, Aisner DL, et al. HER2 Amplification and HER2 Mutation Are Distinct Molecular Targets in Lung Cancers. J Thorac Oncol 2016;11:414-9.

27. Papadimitrakopoulou VA, Wu YL, Han JY, et al. Analysis of resistance mechanisms to osimertinib in patients with EGFR T790M advanced NSCLC from the AURA3 study. Ann Oncol 2018;29:viii741.

28. Ayeni D, Miller B, Kuhlmann A, et al. Tumor regression mediated by oncogene withdrawal or erlotinib stimulates infiltration of inflammatory immune cells in EGFR mutant lung tumors. J Immunother Cancer 2019;7:172.

29. Conforti F, Pala L, Bagnardi V, et al. Sex-Based Heterogeneity in Response to Lung Cancer Immunotherapy: A Systematic Review and Meta-Analysis. J Natl Cancer Inst 2019;111:772-81.

30. Conforti F, Pala L, Bagnardi V, et al. Cancer immunotherapy efficacy and patients' sex: a systematic review and meta-analysis. Lancet Oncol 2018;19:737-46.

31. Smida T, Bruno TC, Stabile LP. Influence of Estrogen on the NSCLC Microenvironment: A Comprehensive Picture and Clinical Implications. Front Oncol 2020;10:137.

32. Hamilton DH, Griner LM, Keller JM, et al. Targeting Estrogen Receptor Signaling with Fulvestrant Enhances Immune and Chemotherapy-Mediated Cytotoxicity of Human Lung Cancer. Clin Cancer Res 2016;22:6204-16.

33. Junttila TT, Li J, Johnston J, et al. Antitumor Efficacy of a Bispecific Antibody That Targets HER2 and Activates T Cells. Cancer Res 2014;74:5561. 